(C) 2018. This manuscript version is made available under the CC-BY-NC-ND 4.0 license http:// creativecommons.org/licenses/by-nc-nd/4.0/

1 Squaring up the health economics of PCSK9 monoclonal antibodies 'down 2 under'

3

\title{
Watts $\mathrm{GF}^{1,2}$, Norman $\mathbf{R}^{3}$.
}

${ }^{1}$ School of Medicine, Faculty of Health and Medical Sciences, University of Western Australia, Perth, Western Australia, Australia

2Lipid Disorders Clinic, Cardiometabolic Services, Department of Cardiology, Royal Perth Hospital, Perth, Western Australia, Australia

${ }^{3}$ School of Public Health, Curtin University, Bentley, Western Australia, Australia

\section{Words: 982}

Seminal data from population genetics have presaged the development of several novel lipid-regulating drugs and the prospect of more effectively addressing high residual risk of cardiovascular disease (CVD) in patients receiving secondary prevention therapies ${ }^{1}$. The most impressive development is the identification of proprotein convertase subtilisin/kexin type 9 (PCSK9), a regulator of the intracellular cycling of the low-density lipoprotein (LDL) receptor, as a target for lowering LDLcholesterol (LDL-C) ${ }^{2}$. This journey most recently entered the phase of large scale CV outcome trials ${ }^{2}$. The most widely tested method for inhibiting PCSK9 entails the use of humanized monoclonal antibodies (mAbs). However, their high acquisition and budgeting impact on healthcare are concerning ${ }^{3}$.

In this issue, Kumar et al report an economic evaluation of PCSK9 inhibitors in Australia ${ }^{4}$. The secondary prevention population relates to the FOURIER trial ${ }^{5}$. Relative to placebo, they estimated an incremental cost-effectiveness ratio (ICER) reporting the cost per quality-adjusted life year (QALY) being higher than $\$ 300,000^{4}$. The authors concluded that a significant reduction in cost is required to reach the point at which PCSK9 inhibitors can be recommended as cost-effective, an appropriate conclusion. There are a number of considerations, both clinical and economic, pointing to future research for demonstrating cost-effectiveness in specific populations.

Several recent studies have considered the cost-effectiveness of PCSK9 mAbs in a range of populations ${ }^{3}$. They have reached similar conclusions to Kumar et al that the high cost of PCSK9 mAbs makes broad use unattractive on cost-effectiveness grounds ${ }^{3,4}$. Importantly, they have also shown that the cost per QALY is sensitive to assumptions made around risk and context. In patients with highest CV risk, one can reasonably expect lower ICERs. Kumar et $\mathrm{al}^{4}$ have also included a useful sensitivity analysis and sub-group analysis exploring the model parameters to which the ICER is most sensitive, fundamentally illustrating the same point; treating patients with a greater CVD risk can significantly reduce the cost per QALY. 
1 A major issue in the evaluation of any risk-lowering agent is high sensitivity to the discount rate ${ }^{6}$. This is an assumption built into economic evaluations accounting for the relative value of costs and outcomes that happen in the future relative to the present ${ }^{6}$. Events in the future are less important than present ones; this has significant implications for interventions which prevent future events. If a $5 \%$ discount rate is used, then the value of costs and outcomes is reduced by that amount for each year into the future. Preventing an event in 10 years' time is worth approximately $40 \%$ less than prevention today. The appropriate discount rate in economic evaluation is uncertain, but Australia tends towards a higher rate $(5 \%$ per annum) than most other countries ${ }^{6}$, implying ceteris paribus relatively less value placed on interventions that prevent future events than other countries do.

The aforementioned analysis ${ }^{4}$ is also underpinned by the results with alirocumab, a comparable PCSK9 mAb, in the ODYSSEY-OUTCOMES trial ${ }^{7}$. In this trial the number of subjects studied was less than FOURIER, but were derived from a postACS population and the duration of intervention was longer. A point of difference with FOURIER, was a nominally significant reduction in total mortality, particularly in patients with LDL-C $>100 \mathrm{mg} / \mathrm{dL}^{7}$, implying greater benefit in patients with higher LDL-C levels².

The impact of baseline LDL-C on the efficacy of PCSK mAbs in reducing CV events is only one factor that bears on cost-effectiveness ${ }^{8}$. Baseline absolute total cardiovascular risk and the degree of absolute or proportional reduction in LDL-C are also key determinants of the number needed to treat (NNT) to prevent a CV event and cost-effectiveness ${ }^{8}$. Duration of therapy is also critical ${ }^{5}$.

Consistent with the above, the FOURIER subgroups that showed greatest absolute risk reduction in CV events, and ipso facto lower NNTs, were patients with diabetes, peripheral arterial disease, recent acute coronary events, recurrent myocardial infarctions and more extensive coronary atherosclerosis ${ }^{2,9}{ }^{9}$. Hence, the costeffectiveness of PCSK9 mAbs is likely to more favourable in these groups but the drug acquisition cost will also need to be decreased ${ }^{3,4}$.

A patient group not included specifically in CV outcome trials of PCSK9 mAb is familial hypercholesterolaemia $(\mathrm{FH})^{10}$. $\mathrm{FH}$ has an extremely high risk of coronary artery disease if untreated. Formerly a challenge, achieving recommended treatment targets of LDL-C is now in FH with the addition of a PCSK9 inhibitor to high potency statin and ezetimibe regimens ${ }^{10}$. Experts recommend PCSK9 mAbs in all patients with $\mathrm{FH}$ who cannot achieve recommended LDL-C treatment targets with standard care, especially if they have symptomatic coronary artery disease, atherosclerosis on imaging or other cardiovascular risk factors, including elevated lipoprotein(a) [Lp(a)] 10.

If the acquisition costs of mAbs cannot be reduced how can we meet the treatment gap? Fortunately, there are other approaches to inhibiting PCSK9 activity, including small interfering RNAs (siRNAs) ${ }^{2}$. Inclisiran, a long-acting siRNA against PCSK9, decreases the production of PCSK9 and the plasma concentrations of LDL-C by an amount that matches that of evolocumab². Supported by preliminary studies, a CV outcome trial with inclisiran will commence shortly². Cheaper manufacturing costs 
1 and longer duration of action imply that if clinical efficacy and long-term safety is 2 confirmed by several studies, RNA interference could become the most cost-

3 effective approach for the inhibition of PCSK9.

4 So what recommendations can we make on the use of PCSK9 mAbs? These agents 5 should unreservedly be reimbursed for all $\mathrm{FH}$ patients far from reaching therapeutic 6 targets of LDL-C despite receiving a high intensity statin and ezetimibe regimen ${ }^{10}$.

7 For other groups of patients in whom LDL-C is not specifically elevated in the $\mathrm{FH}$ 8 range, cost-effectiveness is questionable, as shown in the present and other 9 analyses ${ }^{3,4}$. For these patients, drug acquisition costs should be reduced as soon as 10 possible by a significant proportion and the optimal procurement models and 11 appropriate access schemes negotiated with payers ${ }^{3}$. The price of PCSK8 mAbs needs to be kept well 'down under' the mark and third-party payers need to be also protected by appropriate 'price volume' regulatory policies. 


\section{References}

2 [1] Giugliano, RP and Sabatine, MS, Are PCSK9 Inhibitors the Next

3 Breakthrough in the Cardiovascular Field?, J. Am. Coll. Cardiol., 2015;65:2638-2651.

4 [2] Pirillo, A and Catapano, AL, Proprotein Convertase Subtilisin Kexin 9

5 Inhibitors, Cardiol. Clin., 2018;36:241-256.

6 [3] Hlatky, MA and Kazi, DS, PCSK9 Inhibitors: Economics and Policy, J. Am.

7 Coll. Cardiol., 2017;70:2677-2687.

8 [4] Kumar, R, Tonkin, A, Liew, D, et al., The cost-effectiveness of PCSK9

9 inhibitors-The Australian healthcare perspective, Int. J. Cardiol., 2018.

[5] Sabatine, MS, Giugliano, RP, Keech, AC, et al., Evolocumab and Clinical Outcomes in Patients with Cardiovascular Disease, N. Engl. J. Med., 2017;376:1713-1722.

[6] Attema, AE, Brouwer, WB and Claxton, K, Discounting in Economic Evaluations, Pharmacoeconomics, 2018:in press. Trial: Topline Results - Alirocumab in Patients After Acute Coronary Syndrome, In, American College of Cardiology - 67th Scientific Sessions, 2018. [8] Annemans, L, Packard, CJ, Briggs, A, et al., 'Highest risk-highest benefit'strategy: a pragmatic, cost-effective approach to targeting use of PCSK9 inhibitor therapies, Eur. Heart J., 2017:ehx710.

[9] Bonaca, MP, Nault, P, Giugliano, RP, et al., Low-density lipoprotein cholesterol lowering with evolocumab and outcomes in patients with peripheral artery disease: Insights from the FOURIER trial (Further Cardiovascular Outcomes Research With PCSK9 Inhibition in Subjects With Elevated Risk), Circulation, 2018;137:338-350.

[10] Landmesser, U, Chapman, MJ, Stock, JK, et al., 2017 Update of ESC/EAS Task Force on practical clinical guidance for proprotein convertase subtilisin/kexin type 9 inhibition in patients with atherosclerotic cardiovascular disease or in familial hypercholesterolaemia, Eur. Heart J., 2017:ehx549-ehx549. 